\title{
Spontaneous Regression of Metachronous Intra-Abdominal Desmoid Tumor in a Patient with Familial Adenomatous Polyposis
}

\author{
Kenji Tsuchihashi ${ }^{a}$ Kyoko Yamaguchi ${ }^{a}$ Ryosuke Taguchi ${ }^{a}$ \\ Kenichi Kohashi $^{b}{\text { Kayo } \text { ljichi }^{\mathrm{b}} \text { Yuta Okumura }}^{\mathrm{a}}$ Michitaka Nakano ${ }^{a}$ \\ Akari Ohno $^{c}$ Tomonobu Hiokic Hozumi Shimokawa $^{a}$ Hiroshi Ariyama $^{a}$ \\ Hitoshi Kusaba ${ }^{a}$ Yoshinao Oda $^{\mathrm{b}}$ Koichi Akashi ${ }^{\mathrm{a}}$ Eishi Baba ${ }^{d}$ \\ ${ }^{a}$ Department of Medicine and Biosystemic Science, Faculty of Medical Sciences, Kyushu \\ University, Fukuoka, Japan; 'bepartment of Anatomic Pathology, Graduate School of \\ Medical Sciences, Kyushu University, Fukuoka, Japan; 'Department of Medicine and \\ Bioregulatory Science, Graduate School of Medical Sciences, Kyushu University, Fukuoka, \\ Japan; dDepartment of Oncology and Social Medicine, Graduate School of Medical \\ Sciences, Kyushu University, Fukuoka, Japan
}

\section{Keywords}

Spontaneous regression · Desmoid · Metachronous

\begin{abstract}
Desmoid tumors are clonal fibroblastic neoplasms that arise in soft tissues. Patients with familial adenomatous polyposis (FAP) can develop intra-abdominal desmoid tumors. However, metachronous appearance of intra-abdominal desmoid tumor is rare, and its clinical course is not well known. Here, we report a case of spontaneous regression of metachronous intraabdominal desmoid tumor in a 36-year-old man with FAP. The patient was diagnosed with FAP and underwent laparoscopic total colorectomy. Intra-abdominal desmoid tumor appeared 2 years later and progressed despite treatment with tamoxifen and sulindac. He received four cycles of combinatory therapy with dacarbazine and adriamycin, resulting in shrinkage and stabilization of the desmoid tumor even after cessation of chemotherapy. A new intra-abdominal desmoid tumor developed 2 years later at a different site from the first lesion and progressed from $65 \mathrm{~mm}$ to $70 \mathrm{~mm}$ in diameter within a month. The size of the first lesion, however, remained unchanged. We prepared for chemotherapy because the second lesion progressed, but follow-up computed tomography showed spontaneous shrinkage of the second lesion. The patient
\end{abstract}


still has not needed additional therapy as of more than 4 years after the appearance of the second lesion. Immunohistochemical staining showed the presence of macrophages in the second lesion. Although metachronous intra-abdominal desmoid tumor is rare and management protocols have yet to be established, this case suggests that an active surveillance approach may be applicable under careful follow-up in asymptomatic patients.

(C) 2022 The Author(s).

Published by S. Karger AG, Basel

\section{Introduction}

Desmoid tumors are clonal fibroblastic neoplasms arising in the deep soft tissue. Desmoid tumors grow infiltrative and have a tendency to show local recurrence [1]. The incidence of desmoid tumors is reportedly 2-4 per million. Most desmoid tumors arise sporadically and some are related to trauma, pregnancy, or hereditary cancer syndromes. Approximately, $10-25 \%$ of patients with familial adenomatous polyposis (FAP) develop desmoid tumors [2]. Most desmoid tumors in patients with FAP arise intra-abdominally, representing a main cause of death among FAP patients.

Recently, the Desmoid Tumor Working Group published consensus-based guidelines for dealing with desmoid tumors [3]. Active surveillance is proposed for asymptomatic patients under the supervision of an experienced team. Medical therapies such as surgery, radiotherapy, and/or systemic therapies are considered in patients with symptoms or progressive disease. Intra-abdominal desmoid tumors are close to the gastrointestinal tract and can readily cause intestinal obstruction, ulceration, or bowel ischemia [4]. On the other hand, surgical resection with adequate margins is anatomically impossible, and intra-abdominal desmoid tumors show a strong tendency toward relapsing. Optimal management has thus yet to be defined, particularly for patients with intra-abdominal desmoid tumors.

Desmoid tumors arising metachronously have mainly been reported from extra-abdominal sites [5]. Reports describing metachronous intra-abdominal desmoid tumors are rare, and little is known about the clinical course of this entity. Further, although spontaneous regression can be observed in desmoid tumors, whether metachronous desmoid tumors at intra-abdominal sites can regress spontaneously has not previously been reported. Here, we present a case of spontaneous regression in a FAP patient with metachronous intra-abdominal desmoid tumor.

\section{Case Presentation}

A 36-year-old man with FAP was diagnosed with ascending colon cancer. He underwent laparoscopic total colorectomy, removing an ascending colon cancer with a final stage of T1N0M0 stage I. Follow-up computed tomography (CT) 2 years later showed an intra-abdominal mass and desmoid tumor was suspected (shown in Figure 1a). The patient was started on tamoxifen and sulindac, but the intra-abdominal desmoid tumor progressed (shown in Figure 1b). The pathological diagnosis of desmoid tumor was confirmed from CT-guided biopsy (shown in Figure 1c). Bowel obstruction appeared and insertion of a long intestinal tube was performed. After bowel obstruction resolved, we started combined chemotherapy with dacarbazine and adriamycin (dacarbazine at $150 \mathrm{mg} / \mathrm{m}^{2}$ on days $1-4$ and adriamycin at $20 \mathrm{mg} / \mathrm{m}^{2}$ on days $1-4$ every 4 weeks). The desmoid tumor shrank after four cycles of this regimen. We changed the regimen to tamoxifen and sulindac and continued follow-up monitoring. The desmoid tumor stabilized for 2 years (shown in Figure 1d). Tamoxifen was stopped after the emergence of portal thrombosis. Four months later, follow-up CT showed a new intra-abdominal lesion 


\section{Case Reports in Oncology}

\begin{tabular}{l|l}
\hline \multicolumn{2}{l}{ Case Rep Oncol 2022;15:71-77 } \\
\hline DOI: 10.1159/000521920 & $\begin{array}{l}\text { @ 2022 The Author(s). Published by S. Karger AG, Basel } \\
\text { www.karger.com/cro }\end{array}$ \\
\hline
\end{tabular}

Tsuchihashi et al.: Spontaneous Regression of Metachronous Intra-Abdominal Desmoid Tumor
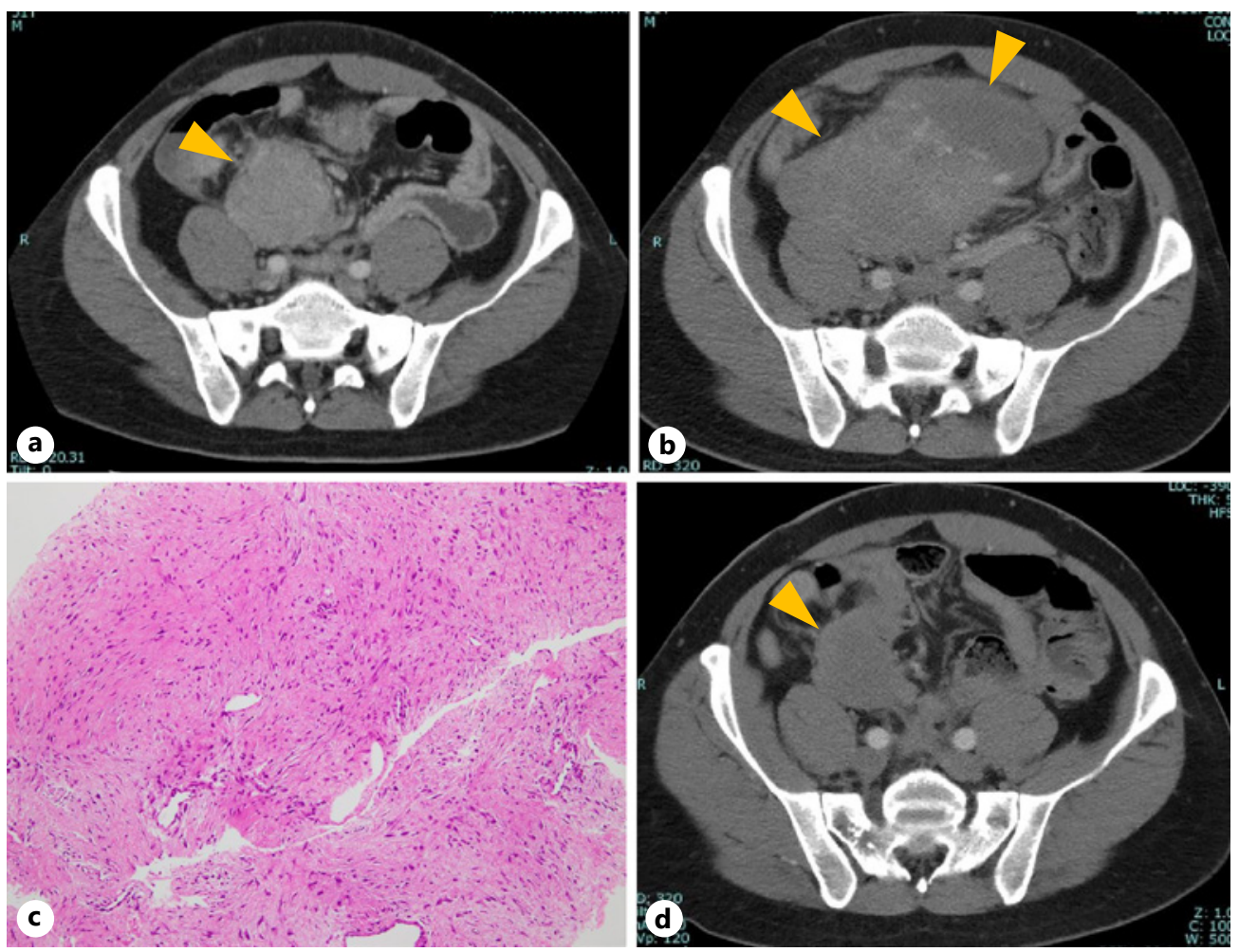

Fig. 1. a CT reveals an intra-abdominal desmoid tumor. b CT shows progression of the intra-abdominal desmoid tumor despite treatment with tamoxifen and sulindac. c HE staining of the intra-abdominal desmoid tumor. $\mathbf{d}$ CT shows the intra-abdominal desmoid tumor has shrunk and stabilized in size after treatment with four cycles of dacarbazine and adriamycin. HE, hematoxylin and eosin.

(diameter, $65 \mathrm{~mm}$ ) in the upper abdomen (shown in Figure 2a). On the other hand, the initial lesion showed no change in size. The new lesion progressed from $65 \mathrm{~mm}$ to $70 \mathrm{~mm}$ within a month. We performed CT-guided biopsy of the new lesion and histopathological examination revealed desmoid tumor (shown in Figure 2b). We prepared to start combined chemotherapy, but the next follow-up CT revealed desmoid tumor had stopped progressing and had started to regress spontaneously. After 4 years from that spontaneous regression was first identified, the second lesion was $41 \mathrm{~mm}$ in diameter and the initial lesion has also remained stable (shown in Figure 2c, d). Recently, we undertook evaluation of immune cells in the desmoid tumor that had shown spontaneous regression. Immunohistochemical analysis of this second lesion showed positive staining for CD68 as the most significant finding. Clear positive staining was also seen for CD163, while CD3, CD8, and CD56 showed weak positive staining. Negative results were seen for CD4 (shown in Figure 3a-f).

\section{Discussion}

The clinical course of metachronous intra-abdominal desmoid tumors is not well understood. We encountered a patient with FAP and metachronous intra-abdominal desmoid tumors. The initial lesion required chemotherapy, but the second lesion regressed spontaneously and has been observed for 4 years.

An aberrant Wnt/APC/ $\beta$-catenin pathway is considered to be the main pathogenesis for desmoid tumor [6]. Most desmoid tumors are sporadic and show mutations in CTNNB1, the 

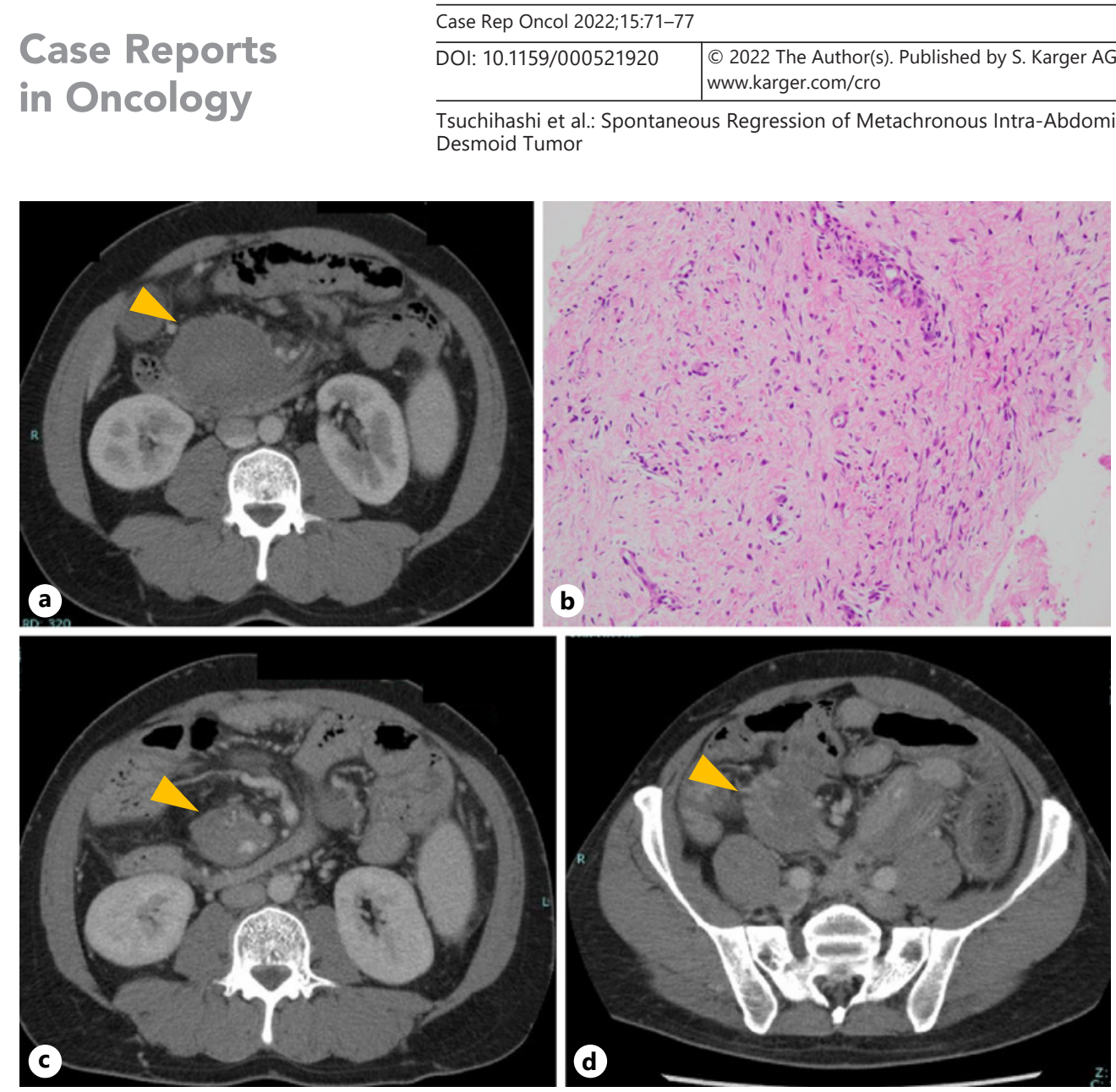

Fig. 2. a CT shows the metachronous intra-abdominal desmoid tumor. b HE staining of the metachronous intra-abdominal desmoid tumor. c CT image of the metachronous intra-abdominal desmoid tumor 4 years after initiation of regression. $\mathbf{d}$ CT shows initial intra-abdominal desmoid tumor at the same time as (c).
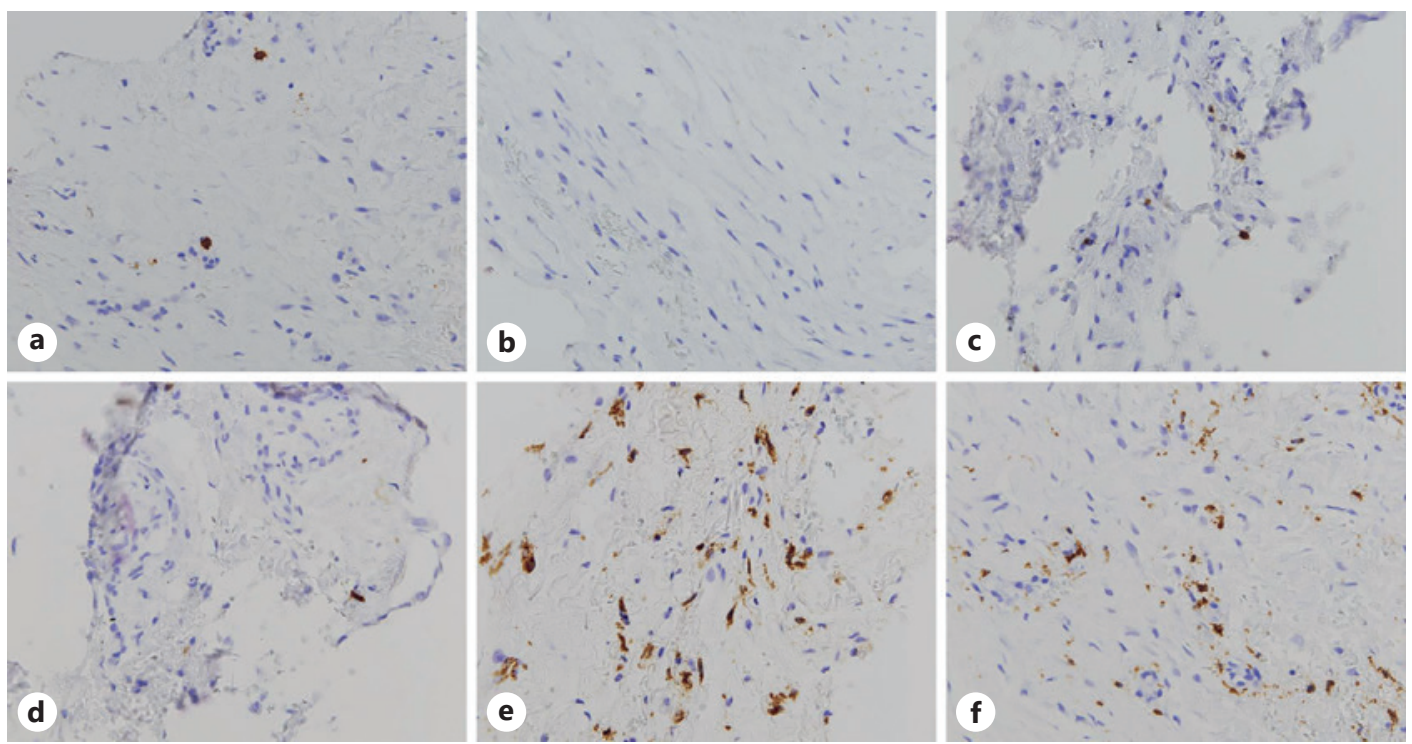

Fig. 3. Immunohistochemical staining of metachronous intra-abdominal desmoid tumor for CD3 (a), CD4 (b), CD8 (c), CD56 (d), CD68 (e), and CD163 (f). 
Tsuchihashi et al.: Spontaneous Regression of Metachronous Intra-Abdominal Desmoid Tumor

gene encoding $\beta$-catenin. Desmoid tumors associated with FAP are often caused by germline mutation in the APC tumor suppressor gene. The incidence of desmoid tumors among FAP patients is $10-25 \%$ [2], an over 800 -fold greater risk compared with the general population [4]. Desmoid tumors in FAP patients tend to occur in the intra-abdominal space, with 50\% arising in this region [7]. A relationship between the incident site and prophylactic surgery for FAP patients has been suggested. Our patient received laparoscopic total colorectomy 2 years prior to the identification of the initial intra-abdominal desmoid tumor. The second intraabdominal desmoid tumor appeared more than 4 months after discontinuation of tamoxifen due to portal thrombosis under treatment with sulindac. The possibility that the cessation of tamoxifen contributed to the development of the second intra-abdominal desmoid tumor cannot be ruled out.

A system for the management of desmoid tumor has been proposed by the Desmoid Tumor Working Group [3]. Asymptomatic patients can be observed initially without active treatment under the supervision of an experienced team. This policy is aimed at avoiding overtreatment since desmoid tumors can stabilize and regress spontaneously. Penel et al. [8] reported that the 2-year event-free survival rate did not differ significantly between patients who underwent surgery and those managed under a wait-and-see approach. Bonvalot et al. [9] reported that $28 \%$ of sporadic desmoid tumors in the abdominal wall showed spontaneous regression. The mechanism underlying spontaneous regression of desmoid tumors is unclear, but initial size $<7 \mathrm{~cm}$ is associated with spontaneous regression. Active treatment is considered for patients with subsequent progression or symptomatic tumor burden. Intra-abdominal desmoid tumors can readily cause intestinal obstruction, ulceration, or bowel ischemia. For this reason, the management of intra-abdominal desmoid tumors is difficult.

Metachronous desmoid tumor has mainly been reported at extra-abdominal sites [5]. Metachronous extra-abdominal desmoid tumors require surgical resection. Reports describing metachronous intra-abdominal desmoid tumors are very rare, and the clinical course is unknown. In the present case, the initial size of the second lesion was $65 \mathrm{~mm}$, subsequently progressing to $70 \mathrm{~mm}$. We were preparing to start combined chemotherapy, but the next follow-up CT revealed this second desmoid tumor had stopped progressing and started to regress spontaneously. Interestingly, the behaviors of the initial lesion and second lesion differed in the present case. Doyen et al. [10] reported nonidentical CTNNB1 mutations in multiple metachronous desmoid tumors. Iwama et al. [11] also reported a recurrent desmoid tumor with a different, additional mutation in $A P C$ in a patient with FAP. Such findings suggest the possibility of differing biology between lesions. Although metachronous intra-abdominal desmoid tumor is rare and its management has not been established, the present case suggests that active surveillance can be applied under careful follow-up in asymptomatic patients.

Siozopoulou et al. [12] reported the status of immune filtration in 33 desmoid tumors. Positive results for CD3 and CD8 were seen in 100\% and 97\%, respectively. Conversely, $27.3 \%$ of samples showed positive results for CD4, and only $9.1 \%$ and $0 \%$ of samples were positive for CD56 and CD68, respectively. The second desmoid tumor in our case was strongly positive for CD68, a marker for macrophages. Macrophages are divisible into pro-inflammatory M1 and anti-inflammatory M2 types [13]. The lesion that regressed also showed positive results for CD163, although to a lesser degree than CD68. CD163 is a marker for M2-like macrophages. On the other hand, Romero et al. [14] reported the presence of CD163 positive macrophage in desmoid-type fibromatosis and its relation to the poor prognosis. A key limitation in the present study was that our specimen is a biopsy sample so that the evaluation of desmoid tumor as a whole is difficult and we were unable to compare immune statuses between the first and second desmoid tumors due to insufficient specimens.

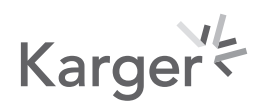




\section{Case Reports in Oncology}

Tsuchihashi et al.: Spontaneous Regression of Metachronous Intra-Abdominal Desmoid Tumor

\section{Conclusion}

In summary, we encountered a case in which metachronous intra-abdominal desmoid tumor showed spontaneous regression in a patient with FAP. A standard approach for treating metachronous desmoid tumors has not yet been established. Active surveillance appears applicable for metachronous desmoid tumors under careful monitoring if the lesions remain asymptomatic.

\section{Acknowledgment}

We would like to thank Masami Obuchi for the assistance to prepare the manuscript.

\section{Statement of Ethics}

Written informed consent was obtained from the patient for publication of the details of medical information including images. Case reports are granted an exemption from requiring ethical approval at Kyushu University Hospital.

\section{Conflict of Interest Statement}

The authors have no conflicts of interest to declare.

\section{Funding Sources}

The authors did not receive any funding for this study.

\section{Author Contributions}

K.T. and E.B. contributed the design of the report and drafted the manuscript. K.K., K.I., and Yoshinao O. interpreted the histological slides. K.Y., R.T., Yuta O., M.N., A.O., T.H., H.S., H.A., H.K., and K.A. contributed to interpretation of data and critically revised the manuscript.

\section{Data Availability Statement}

All data generated or analyzed during this study are included in this article. Further inquiries can be directed to the corresponding author.

\section{References}

1 Escobar C, Munker R, Thomas JO, Li BD, Burton GV. Update on desmoid tumors. Ann Oncol. 2012 Mar;23(3): 562-9.

2 Sturt NJ, Clark SK. Current ideas in desmoid tumours. Fam Cancer. 2006;5(3):275-8; discussion 287-8.

3 Alman B, Attia S, Baumgarten C, Benson C, Blay J-Y, Bonvalot S, et al. The management of desmoid tumours: a joint global consensus-based guideline approach for adult and paediatric patients. Eur J Cancer. 2020 Mar 1; 127:96-107.

\section{Karger's}




\section{Case Reports in Oncology}

\begin{tabular}{l|l}
\hline Case Rep Oncol 2022;15:71-77 \\
\hline DOI: 10.1159/000521920 & $\begin{array}{l}\text { @ 2022 The Author(s). Published by S. Karger AG, Basel } \\
\text { www.karger.com/cro }\end{array}$ \\
\hline
\end{tabular}

Tsuchihashi et al.: Spontaneous Regression of Metachronous Intra-Abdominal Desmoid Tumor

4 Desurmont T, Lefèvre JH, Shields C, Colas C, Tiret E, Parc Y. Desmoid tumour in familial adenomatous polyposis patients: responses to treatments. Fam Cancer. 2015 Mar;14(1):31-9.

5 Mankin HJ, Hornicek FJ, Springfield DS. Extra-abdominal desmoid tumors: a report of 234 cases. J Surg Oncol. 2010 Oct 1;102(5):380-4.

6 Penel N, Chibon F, Salas S. Adult desmoid tumors: biology, management and ongoing trials. Curr Opin Oncol. 2017 Jul;29(4):268-74.

7 Clark SK, Neale KF, Landgrebe JC, Phillips RK. Desmoid tumours complicating familial adenomatous polyposis. Br J Surg. 1999 Sep;86(9):1185-9.

8 Penel N, Le Cesne A, Bonvalot S, Giraud A, Bompas E, Rios M, et al. Surgical versus non-surgical approach in primary desmoid-type fibromatosis patients: a nationwide prospective cohort from the French Sarcoma Group. Eur J Cancer. 2017 Sep;83:125-31.

9 Bonvalot S, Ternès N, Fiore M, Bitsakou G, Colombo C, Honoré C, et al. Spontaneous regression of primary abdominal wall desmoid tumors: more common than previously thought. Ann Surg Oncol. 2013 Dec;20(13): 4096-102.

10 Doyen J, Duranton-Tanneur V, Hostein I, Karanian-Philippe M, Chevreau C, Breibach F, et al. Spatio-temporal genetic heterogeneity of CTNNB1 mutations in sporadic desmoid type fibromatosis lesions. Virchows Arch. 2016 Mar;468(3):369-74.

11 Iwama T, Kuwabara K, Ushiama M, Yoshida T, Sugano K, Ishida H. Identification of somatic APC mutations in recurrent desmoid tumors in a patient with familial adenomatous polyposis to determine actual recurrence of the original tumor or de novo occurrence. Fam Cancer. 2009;8(1):51.

12 Siozopoulou V, Marcq E, Jacobs J, Zwaenepoel K, Hermans C, Brauns J, et al. Desmoid tumors display a strong immune infiltration at the tumor margins and no PD-L1-driven immune suppression. Cancer Immunol Immunother. 2019 0ct;68(10):1573-83.

13 Jayasingam SD, Citartan M, Thang TH, Mat Zin AA, Ang KC, Ch'ng ES. Evaluating the polarization of tumorassociated macrophages into M1 and M2 phenotypes in human cancer tissue: technicalities and challenges in routine clinical practice. Front Oncol. 2019;9:1512.

14 Romero S, Szafranska J, Cabrera E, Gonzalez A, Peiró A, Llauger J, et al. Role of tumor-associated macrophages and angiogenesis in desmoid-type fibromatosis. Virchows Arch. 2012 Aug;461(2):117-22. 